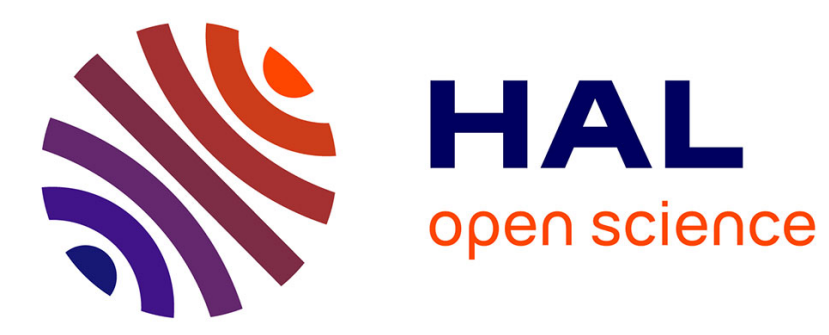

\title{
La montagne cosmique dans les mondes indien et tibétain : mont Meru et mont Kailas \\ Gérard Toffin
}

\section{To cite this version:}

Gérard Toffin. La montagne cosmique dans les mondes indien et tibétain : mont Meru et mont Kailas. Le monde alpin et rhodanien, 1988, La haute montagne. Visions et représentations de l'époque médiévale à 1860, 16 (1), pp.13-29. 10.3406/mar.1988.1355 . hal-00589619

\section{HAL Id: hal-00589619 https://hal.science/hal-00589619}

Submitted on 15 Feb 2018

HAL is a multi-disciplinary open access archive for the deposit and dissemination of scientific research documents, whether they are published or not. The documents may come from teaching and research institutions in France or abroad, or from public or private research centers.
L'archive ouverte pluridisciplinaire HAL, est destinée au dépôt et à la diffusion de documents scientifiques de niveau recherche, publiés ou non, émanant des établissements d'enseignement et de recherche français ou étrangers, des laboratoires publics ou privés. 


\title{
La montagne cosmique dans les mondes indien et tibétain : mont Meru et mont Kailas
}

\author{
Gérard Toffin
}

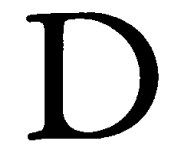

ANS un livre récent consacré à l'architecture et aux jardins miniatures d'Extrême-Orient, Rolf $A$. Stein analyse en détail les relations réciproques entre réel et symbolique dans la pensée religieuse asiatique ${ }^{(1)}$. Pour lui, traits réels de l'architecture et spéculations religieuses ne s'excluent pas : il convient d'étudier les deux de pair, de reconnaître les transformations par lesquelles la pensée symbolique se distingue de la conscience ordinaire, tout en cherchant l'origine de certaines cosmogonies dans le plan des habitations primitives. L'imagination, affirme-t-il, est souveraine mais les symboles les plus élaborés s'ancrent souvent dans des faits matériels, l'habitation par exemple. Cette prise de position renvoie ou s'efforce de renvoyer dos à dos les théories dites empiristes qui considèrent le symbole comme un reflet, plus ou moins déformé, d'une expérience sensorielle, et celles plus intellectualistes qui insistent sur le caractère arbitraire du signe linguistique, trait consubstantiel du symbolique, antérieur à toute action de l'homme sur la nature. R. A. Stein semble s'attacher à une voie moyenne, dialectique, qui prendrait en compte aussi bien la part d'imaginaire dans le réel que la part de réel dans l'imaginaire.

Le registre « naturel » (plantes, animaux, phénomènes naturels) joue un rôle aussi important dans la pensée symbolique, peut-être plus, que le registre " architectural ». Les deux codes apparaissent d'ailleurs souvent confondus dans les religions asiatiques, de multiples homologies permettant de passer d'un registre à l'autre. La nature est présente métaphoriquement dans toute culture; elle y influe sur un mode qui n'est pas celui de la simple causalité, mais qui n'entraîne pas moins, sous certains rapports, des chaînes causales. Ici aussi, il est possible, au moins théoriquement, de défendre une voie médiane, à égale distance du réductionnisme géographique élémentaire qui assigne aux sociétés humaines des déterminations écologiques, et des théories culturalistes qui privilégient la structuration de la réalité environnementale par notre imaginaire. Toute civilisation religieuse d'Extrême-Orient, Paris, Flammarion, 1987. 
procède de la nature autant que de la culture soutient par exemple Augustin Berque dans son ouvrage sur le sentiment de la nature au Japon, car l'homme participe des deux : "Lidéel ne va pas sans matériel et à l'inverse le matériel ne va pas sans idéel » ${ }^{(2)}$. Claude Lévi-Strauss lui-même, dont on connaît le rationalisme intransigeant, écrit dans Le Regard éloigné qu' « en essayant de connaître le monde, l'esprit accomplit des opérations qui ne diffèrent pas en nature de celles qui se déroulent dans le monde depuis le commencement des temps ${ }^{(3)}$. Ces prises de position rapprochées, qui recouvrent quelque chose de très profond dans les réflexions contemporaines, mettent en question les vieux ancrages doctrinaux et les lignes de démarcation trop tranchées. Elles font souffler un vent frais sur des questions jusqu'ici présentées comme des apories.

En matière de symbolisme, l'important est que le substrat matériel soit «bon à penser », qu'il soit sociologiquement et religieusement exploitable. La haute montagne justement, fait partie de ces éléments physiques. Son aspect vertical et insolite (vu de la plaine), sa végétation caractéristique, sa couverture de neige et de glace, sa puissance immobile, sa proximité de la voûte céleste qui engendre le feu (la foudre), l'ont en quelque sorte prédestinée à la sacralisation. De par leurs cimes, les hautes montagnes appartiennent à cette sphère suprahumaine où gravitent les étoiles et les astres. Quand elles ne sont pas divinisées elles servent de résidence terrestre aux divinités de l'En-Haut. Tout se passe comme si les sociétés humaines avaient d'autant plus chargé de représentations ces sommets qu'elles avaient été sensibles à l'aura numineuse qui s'en dégage - de la même manière que certains symboles fondamentaux, peut-être encore plus fondamentaux que ceux évoqués ici, dérivent de schémas corporels et d'oppositions centrées sur le corps. Cette dimension religieuse de la haute montagne a été reconnue par toutes les sociétés et l'on reste songeur devant la portée quasi universelle des images élémentaires qui lui sont associées : pilier du ciel, centre de l'univers, pont reliant les différents mondes cosmiques, etc. Reste que chaque culture attribue un sens particulier à cette montagne qui lui est propre. Reste également que la fonction symbolique permet de dépasser les données du réel et dévoile une structure du monde située au-delà de l'expérience immédiate: le symbolisme se définit en dernière analyse comme un système de valeurs où chaque élément est doté d'une signification qui ne saurait être déterminée mécaniquement par la propriété physique de ce qu'il désigne.

On se propose de contribuer ici à une meilleure connaissance du symbolisme de la montagne en analysant les principales représentations attachées au Mont cosmique dans les mondes indien et tibétain. On partira du modèle imaginaire original : le mont Meru, tel que le décrivent les textes indiens les plus anciens. Ce modèle est essentiel car il fut diffusé dans une grande partie de l'Asie dès le début de l'ère chrétienne, sans doute même avant. On s'attachera ensuite aux identifications physiques, aux matérialisations de cette montagne mythique, en prenant l'exemple d'un des sommet

(2) A. BERQUE, Le sauvage et l'artifice. Les Japonais devant la nature, Paris, Gallimard, 1986, p. 128.

(3) C. Lévi-Strauss, Le Regard éloigné, Paris, Plon, 1983, pp. 164-165. 
les plus sacrés de l'Himalaya : le mont Kailas. Il s'agira de faire comprendre quelques-unes des idées anciennes, quoique toujours vivantes, auxquelles la haute montagne est associée dans cette partie du monde. Cette confrontation entre modèle imaginaire et figurations naturelles sera l'occasion de souligner les liens étroits entre matériel et symbolique dans la pensée religieuse asiatique.

\section{LE MONT MERU DANS LA COSMOLOGIE HINDOUE ET BOUDDHISTE}

A l'origine, disent les hymnes védiques, il n'y avait ni jour ni nuit, ni ciel ni terre, ni temps ni être. Plongé dans des ténèbres indistinctes, le monde formait une unité indifférenciée, incoordonnée (asat : « non être »). Seules les Eaux (âpas), porteuses d'un germe de vie, existaient, des eaux sans limites et d'une profondeur insondable. Un moment vint - sans intervention divine d'aucune sorte - où monta du fond de ces Eaux originelles un petit morceau de terre qui vint surnager à la surface de l'Océan primordial. Cette motte de terre grandit, devint une montagne et flotta à la surface des flots, à la dérive( ${ }^{(4)}$.

C'est alors qu'Indra, le roi des dieux, qui préexistait semble-t-il à la création du monde, intervient. Il affermit cette montagne, l'immobilisa définitivement en tuant un démon appelé $V$ ṛta, symbole des forces d'obstruction et de résistance, opposé au monde ordonné et habitable. Vrrta, décrit parfois sous la forme d'un serpent, résidait à l'intérieur de la Montagne primitive ; il bloquait les eaux et les fleuves qui y étaient contenus et fermait l'espace entre Terre et Ciel. Indra le tua en ouvrant la Montagne et en écrasant la tête du démon avec son arme en forme de foudre, le vajra. Vrta mort, la Terre se trouva fixée solidement et les Eaux, symbole de vie, s'écoulèrent librement : elle surgirent du sommet du pic et se déversèrent des quatre côtés de l'éminence, filant vers l'océan dans les quatre directions de l'univers.

La Montagne une fois fixée continua de croître. Elle constitua progressivement ce qui est aujourd'hui la Terre. Elle en devint le point d'ancrage, l'axe primordial. Le Mont cosmique exhaussa également le Ciel, le plaçant à sa place actuelle. Cette dissociation du Ciel et de la Terre, à laquelle Indra participa activement, est un élément déterminant de la genèse du monde; elle parachève la formation d'un univers différencié, ordonné, sat (« être »), habitable, où les éléments autrefois confondus se trouvent distingués. Ce processus s'accompagne de la création de l'aube et

(4) On s'est principalement basé ici sur l'ouvrage de F. B. KUIPER, Ancient Indian cosmogony, Delhi, Vikas Publishing House, 1983, qui expose avec beaucoup de force les faits relatifs à la Montagne cosmique dans les hymnes védiques. Ces textes sont cependant loin d'être clairs : ils admettent des variantes, voire des interprétations différentes. $A$ ce sujet, voir F. D. K. Bosch, The golden Germ. An introduction to Indian symbolism, 'S-Gravenhage, Mouton \& $\mathrm{Co}, 1960 ; \mathrm{N}$. BROWN, "The Creation myth of the Rig Veda", Journal of the American Oriental Society, 1942, vol. 62, pp. 85-98; J. GONDA, Les Religions de l'Inde; I - Védisme et Hindoussme ancien, Paris, Payot, 1962 ; A. M. EsNOUL, " La naissance du monde dans l'Inde », in La Naissance du monde, Paris, Ed. du Seuil (Col. «Sources orientales $\gg)$, 1959, pp. 329-365. 
de la division du temps en une phase diurne et une phase nocturne. On retiendra les aspects génésiques du mythe : l'apparition de la Montagne surgissant de l'horizon indistinct et de l'Océan primordial coïncide avec l'apparition de la vie, elle instaure une première organisation de la matière inerte. On remarquera également la place inaugurale dévolue à la notion de centre. Face à un monde incohérent, menaçant, incompréhensible, le premier souci de l'homme est de déterminer un point d'ordonnancement central, un omphalos, d'où tout serait sorti et autour duquel l'univers tout entier s'oriente.

Les textes purâniques, postérieurs de plusieurs siècles aux Véda(5), fournissent des indications supplémentaires sur la configuration de l'univers $^{(6)}$. La Montagne primitive dont l'émergence vient d'être relatée reçoit un nom : Meru, Mahâmeru ou encore Sumeru. Elle occupe le centre du monde et commande l'ordonnance géographique de l'univers. Toutes les constellations tournent autour d'elle. Sa couleur est dorée ; elle brille d'un éclat encore plus éblouissant que le soleil. Le Vișnu Purâna qui consacre un chapitre entier à la description de la Terre, indique qu'au sommet de ce mont pousse l'arbre jambu, un pommier rose $\mathrm{e}^{(7)}$, aux fruits gros comme des éléphants. C'est là qu'est bâtie la demeure du dieu Brahmâ (Brahmâloka), créateur de l'univers, flanquée par les cités des huit gardiens de l'espace, Aștadikpâla. Ce sommet, auquel une majestueuse allée d'étoiles conduit, est pourvu de richesses sans pareilles : il est couvert de plantes et d'essences odoriférantes. Les âmes des morts y attendent de futures renaissances. La montagne a 84000 lieues (yojana) de hauteur visible et s'enfonce à 16000 lieues sous terre ${ }^{(8)}$. Elle est plus large à son sommet qu'à sa base. Les Purâna expliquent de manière poétique que la Terre est un lotus dont le Meru forme le péricarpe.

Reprenant la vieille image védique, le Viṣnu Purâna (II,2) dit également que la rivière Gangâ, née du pied de Viṣnu, fait le tour de la cité de Brahmâ, au sommet du Meru, puis se divise en quatre fleuves (nadî) qui se fraient un chemin dans les quatre directions de l'univers, purifiant tous les péchés sur leur passage, et finissent par se jeter dans l'océan. Ces quatre fleuves ont pour nom : Sîtâ, qui se dirige vers l'est et le pays Bhadraśva : Alakanandâ [c'est le nom aujourd'hui du principal affluent himalayen du Gange], qui se dirige vers le sud et le pays Bhârata ; Chakșu, qui conduit vers l'ouest et le pays Ketumâla ; Bhadrâ, lui, mène vers le nord et le pays Uttarakuru. Le Viṣnu Purâna [II, 2) fait état par ailleurs de quatre montagnes sacrées autour du Meru: Mandara, Gandhamâdana, Vipula et Supârsva. Quatre arbres particuliers, dressés comme des tours et hauts chacun de 1100 lieues (yojana), pousseraient à leur sommet. Ces essences ont pour nom:

(5) Les Purâna, énormes compilations de mythes et de cosmogonies, ont été composés à partir des premiers siècles de l'ère chrétienne jusqu'au XII e siècle, parfois au-delà.

(6) On laissera ici de côté les aspects relatif́s au monde sacrificiel dans les cosmologies purâniques. Pour plus de détails sur ce sujet, cf. A. M. ESNOUL, op. cit., et M. BIARDEAU, Etudes de mythologie hindoue. T. I.: Cosmogonies purâniques, Paris, Ecole française d'Extrême-Orient (vol. CXXVIII), 1981.

(7) Eugenia jambolana, d'après M. \& J. STULEY, A Dictionary of Hinduism, London, Routledge \& Kegan Paul, 1977, p. 125.

(8) Mârkaṇdeya Purâna, 54, 15f, et Viṣnu Purâna, II, 2. 
Anthocephallus cadamba (sanskrit: kadamba), Eugenia jambolana (ou Zyzgium cumini) (skt.: jambu), Ficus religiosa (skt.: pippala), Ficus bengalensis (skt.: vata).

Divers continents, appelés $d v \hat{z} p a$ (« îles »), se trouvent disposés autour de la Montagne cosmique et de ses contreforts. Deux conceptions prévalent à cet égard. Dans certains textes (Mahâbhârata), il est question de quatre continents placés aux points cardinaux. Celui qu'habitent les hommes se nomme Jambudvîpa («continent de la pomme rose »), terme qui désigne plus spécifiquement l'Inde ; il est situé au sud. Au nord du Meru s'étend un pays fabuleux : l'Uttarakuru. A l'ouest et à l'est sont localisées deux autres étendues terrestres dont on sait peu de choses. Dans d'autres textes, purâniques notamment, le mont Meru est entouré de sept continents concentriques séparés les uns des autres par des océans eux aussi en forme d'anneaux et composés de différentes substances : eau salée, jus de canne à sucre, vin, beurre fondu, lait caillé, lait, eau douce, synonymes de fertilité et d'abondance. Le premier continent, celui au centre duquel s'élève le mont Meru, est le Jambudvîpa. Lui seul est habité par des hommes. Il compte six chaînes de montagne (parvata) parallèles, orientées d'est en ouest. La chaîne la plus méridionale est l'Himavant, autrement dit l'Himalaya. Le Meru se dresse dit-on au milieu de ces montagnes, entre la troisième et la quatrième chaîne. Ces six massifs montagneux délimitent sept zones (varșa : versant, littéralement " pluie »), dont la plus septentrionale est l'Uttarakuru, et la plus méridionale : le Bhârata, le versant des Bhârata, c'est-à-dire l'Inde. Ce dernier pays est la terre du karma, karmabbâmi: les hommes y souffrent, ils y sont assujettis au cycle des renaissances infinies, mais c'est uniquement là qu'ils peuvent accéder à des états supérieurs. Les autres contrées sont des terres de jouissance, des pays de Cocagne; leurs habitants ignorent la succession des quatres ères (yuga), ils vivent très vieux et sont réputés pour leurs qualités physiques surprenantes. Au niveau du septième océan, l'univers est ceinturé par une muraille rocheuse périphérique $^{(9)}$.

A cette représentation horizontale s'ajoute un symbolisme vertical. Le mont Meru relie le monde souterrain au monde terrestre, où vivent les hommes, et au ciel, domaine des dieux. L'univers, est-il imaginé, consiste en sept couches superposées, la plus haute étant la résidence céleste de Brahmâ.

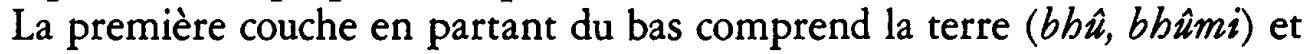
les mondes inférieurs (pâtâla), lesquels consistent à leur tour en sept enfers (naraka). Dans le dernier des mondes inférieurs vit le serpent Vâsuki qui supporte le mont Meru et les divers mondes grâce à son capuchon de cobra largement déployé. Vâsuki menace constamment l'univers ; ses bâillements provoquent des tremblements de terre et lorsqu'il se réveille en déroulant son corps gigantesque une ère cosmique, $k a l p a$, s'achève : l'univers tout entier est consumé par son souffle terrifiant.

(9) Sur cette représentation du monde, voir L. RENOU et J. FILLIOZAT, L'Inde classique, Paris, Payot, 1947, tome I, pp. 547-548, et J. GoNDA, op. cit., pp. 393-394. Une des sources de base est le Viṣnu Purâna, Ed. \& translated by H. H. WILSON, The Viṣnu Purâna. A System of Hindu mytbology and tradition. Calcutta, Punthi Pustak, 1961 (1 édit. : 1940). 
Une autre montagne cosmique a son importance dans la mythologie hindoue et mérite d'être mentionnée ici. Il s'agit du mont Mandara, une montagne imaginaire, située vers l'est et considérée comme une forme du Meru. Le Mandara est connu pour avoir servi de batteur lors du barattage de l'Océan de lait. Cet épisode mythique se rattache à la légende du déluge. Lorsque les Eaux montèrent et envahirent toute la terre, l'ambroisie, amrta, breuvage grâce auquel les dieux préservaient leur jeunesse, fut perdue. Le dieu Viṣnu délégua sur terre un de ses avatars: la Tortue Kurma, la deuxième dans la série de ses incarnations, en la chargeant de retrouver le breuvage magique. La Tortue, dans l'affaire, servit de support à la Terre et au mont Mandara. Vâsuki, prince des serpents, fut disposé comme une corde autour de la montagne, puis dieux, deva, et démons, asura, tirant à hue et à dia sur les deux extrémités du Dieu-serpent, imprimèrent au Mandara un mouvement tournant. De ce long barattage naquirent l'ambroisie ainsi que plusieurs éléments merveilleux : la lune, la déesse Srî, Surâ (une boisson enivrante), un cheval blanc, la pierre précieuse kaustubba qui orne la poitrine de Viṣnu, l'éléphant Airâvaṇa dont Indra prit possession, l'arbre céleste pârijâta, la vache d'abondance Surabhi, les nymphes Apsara et un poison. Comme nous le verrons plus loin, ce mouvement rotatif est un aspect non négligeable du symbolisme de la Montagne sacrée dans les religions asiatiques ${ }^{(10)}$.

La cosmologie bouddhique s'apparente étroitement aux représentations hindoues. Elle repose elle aussi sur l'idée d'une montagne centrale, le Meru (ou Sumeru) [en tibétain Ri-rab], axe de l'univers au-dessus duquel s'étagent les divers cieux, et moyen de communication entre Terre et Ciel. Cette montagne est entourée par sept chaînes orographiques concentriques séparées par autant de mers. Autour de ce complexe s'étend le grand Océan dans lequel baignent les quatre continents insulaires, et leurs satellites, un dans chaque direction de l'univers. Les continents ont une base dorée en forme de tortue. Le Jambudvîpa, seul pays habité, est situé au sud, comme dans le modèle hindou: les autres continents sont «mythiques».

$\mathrm{Au}$ sommet du mont Meru se dresse la résidence des quatre régents des points cardinaux. Au-dessus encore trône Indra, entouré des Trente-trois dieux. Il est également rapporté que le mont Meru est couvert de pierreries. Ses faces sont chacune d'une matière précieuse particulière : la face Est est en cristal (ou en argent), la face Sud en saphir ou en lapis lazuli, celle de l'Ouest en rubis, celle du Nord en or. Les textes bouddhiques ont également la notion d'une enceinte clôturant l'ensemble de l'univers; cette double muraille de fer est appelée cakravâla (11).

Dans les sources chinoises, le Sumeru bouddhiste est parfois figuré comme un cône pointu ćmcrgcant de la mer ct portant à son sommet un

(10) Mentionnons également le mont Govardhana, autre montagne imaginaire, importante dans la mythologie hindoue. Liè à Krșna, il mérite une étude spéciale.

(11) Sur la conception bouddhiste de l'univers, voir par exemple A. WADDELL, Buddhism and Lamaism of Tibet, New Delhi, Heritage Publishers, 1974 (1re édit. : 1895) ; J. FILlIOZAT, "Le symbolisme du monument du Phnom Bähèn », Bulletin de l'Ecole Française d'Extrême-Orient, 1954, vol. XLIV, pp. 527-554 ; G. COEDES, Pour mieux comprendre Angkor, Paris, Adrien Maisonneuve, 1947 ; R. STEIN, op. cit. 
autre cône renversé qui représente la demeure des Trente-trois dieux dont Indra est le chef. L'ensemble fait penser à un sablier : large en haut et en bas, le mont serait resserré au milieu. C'est là, peut-on penser, que s'enroulèrent les deux cordes en forme de serpent qui servirent à faire tourner le mont Mandara (=Sumeru) autour de son axe dans le mythe indien du barattement - mythe largement diffusé en Asie centrale, au Tibet et en Extrême-Orient. Les données qu'a rassemblées récemment $R$. A. Stein attestent que le Sumeru n'est pas seulement ce pic immobile, inébranlable qu'on s'imagine habituellement, mais qu'il est aussi animé d'un mouvement tournant toujours implicite. Cette notion apparaît clairement dans divers motifs architecturaux qui représentent le Sumeru sous forme de temple-montagnes ou de piliers ${ }^{(12)}$.

Les conceptions tibétaines reposent sur des croyances autochtones très anciennes auxquelles se sont superposées les idées tirées des écritures bouddhiques. La Montagne sacrée, ici comme ailleurs, est imaginée comme un immense rocher, une échelle permettant d'accéder au Ciel. Les Tibétains y voient le support de la voûte céleste et le siège des Bouddha. Ils lui donnent l'épithète de "Clou de la Terre », sa'yi phurbu, car le Sumeru fixe la surface terrestre et empêche les démons de surgir des domaines souterrains ${ }^{(13)}$. On retrouve par ailleurs dans ce pays le thème des Eaux nourricières et primordiales. Le plateau tibétain, dit-on, était autrefois couvert par un vaste lac. En ouvrant une brèche dans l'Himalaya pour laisser passer le Tsangpo, un bodhisattva aurait vidé cette étendue d'eau et rendu le pays habitable ${ }^{(14)}$. La Terre, immobile, est conçue comme un lotus à huit pétales, tandis que le Ciel, perpétuellement en mouvement, est décrit comme une roue à huit raies.

Les représentations associées à la haute montagne dans les textes hindous et bouddhistes révèlent donc un symbolisme foisonnant. La Montagne cosmique ne peut être réduite à une seule idée, elle exprime une série complexe de notions. Schématiquement, le mont Meru est à la fois :

- la source générique de l'univers, tout particulièrement de la Terre;

- l'axe central d'un univers clos, circonscrit à ses extrémités;

- le soutien de la voûte céleste et le pilier, parfois animé d'un mouvement tournant, assurant la cohésion des trois mondes superposés;

- un moyen de communication entre Ciel et Terre, un trait d'union qui peut être emprunté dans un sens comme dans un autre par certaines énergies ;

- il recèle en son centre une force obscure, démoniaque, qu'il convient de vaincre pour établir ou maintenir la prospérité.

Ces images constituent le prototype de croyances qui, à quelques variantes près, se retrouvent dans une large partie de l'Asie, du Japon à Java, de la Birmanie au Cambodge. Elles ont joué un rôle essentiel dans l'art et

(12) R. A. STEIN, op. cit.

(13) Ibid., p.196.

(14) Ce thème existe ailleurs en Himalaya. Pour la vallée de Kathmandou, cf. G. ToFfiN, Société et religion chez les Néwar du Népal. Paris, Ed. du CNRS, 1984, pp. 51-53. 
l'architecture. Le stûpa de Sanchi, en Inde centrale, construit au IIIe siècle avant notre ère, le monastère de Samye, le plus ancien au Tibet, dans le bas Tsangpo, édifié au VIII siècle après J.-C., le stûpa de Borobodur à Java, construit au IXe siècle après J.-C., ont tous été bâtis sur ce modèle. Ils symbolisent le pilier central de l'univers, surplombant quatre continents localisés aux quatre points cardinaux. Ce sont des monts Meru à échelle réduite, des cosmogrammes gravés dans la pierre et exposés à la méditation des fidèles. En reproduisant le macrocosme au niveau du microcosme, en établissant des correspondances entre l'un et l'autre, les architectes et prêtres asiatiques cherchaient à établir une harmonie bénéfique entre l'univers et le monde des humains. Une telle « cosmisation » consacrait par ailleurs le monument religieux et l'établissait définitivement comme espace sacré.

Les mêmes idées, le même symbolisme imprègnent de nombreux aspects de la vie religieuse hindoue et bouddhiste. C'est le cas du linga, représentation phallique du dieu Śiva et symbole de l'énergie cosmique, du pilier central de la maison auquel on rend un culte particulier, et du poteau sacrificiel auquel on attache des bêtes destinées à être offertes aux dieux. Tous ces éléments verticaux sont assimilés peu ou prou à l'axis mundi de l'univers, générateur de vie, donc au mont Meru. De la même manière, la cité bouddhique, avec son palais royal au centre et ses quatre stûpa à la périphérie, reproduit ces notions : elle garantit au roi, doublet terrestre d'Indra, une place focale dans le royaume et fait explicitement de lui un trait d'union entre la Terre et le Ciel. Les diagrammes magiques, mandala et yantra, qui représentent le monde en petit et que les prêtres utilisent dans les rituels, sont eux aussi organisés autour d'un centre, symbole de l'absolu. Lorsqu'il s'aide de ces diagrammes dans sa méditation, le disciple tantrique et yogique doit identifier sa colonne vertébrale au mont Meru. Il fait remonter la force brûlante kundalinî (présente dans chaque être humain sous la forme d'un serpent lové à la base du tronc) le long de son épine dorsale jusqu'au sommet de la tête. Là, ce feu fait fondre une goutte qui en redescendant le long de la colonne devient un flot d'ambroisie et provoque un état de félicité totale. Le disciple parvient ainsi à se dissoudre dans l'univers ; il retrouve l'unité primitive disparue et transcende les oppositions contingentes entre Terre et Eau, sexe masculin et sexe féminin, lumière et obscurité. L'efficacité de ce symbolisme repose sur l'idée que le corps humain est organisé selon les mêmes principes que le cosmos, et que la connaissance de l'un implique la connaissance de l'autre.

Il est vraisemblable que ces représentations ont leur source, au moins en partie, dans la mythologie hindoue. La civilisation indienne a rayonné dans la majeure partie de l'Asie orientale dès le deuxième siècle avant notre ère, soit directement, soit par bouddhisme interposé, et a exercé un rôle considérable dans les domaines artistiques et religieux partout où elle pénétrait. Comme l'écrit R. A. Stein : « Depuis l'arrivée du bouddhisme, des images indiennes ont été couramment adoptées et ont augmenté le lot du langage architectural et cosmographique propre à la Chine et au Tibet, au Japon et à l'Asie du Sud-Est »(15). Ces images se sont mêlées ici et là à des

(15) R. A. STEIN, op. cit., p. 230. 
croyances autochtones, d'où les formes parfois légèrement différentes prises par le culte aux montagnes selon les pays.

Il est également plus que probable que les idées cosmographiques indiennes s'inspirèrent de la chaîne himalayenne qui, sur près de $3000 \mathrm{~km}$, barre l'horizon septentrional du sous-continent. La simple vue de cette barrière couverte de neiges éternelles comporte en soi quelque chose de spectaculaire pour l'homme des plaines. Sans verser dans un déterminisme réducteur, il n'y a guère de raison de mettre en doute une relation entre fait géographique et représentation symbolique sur ce point. Un tel passage du concret à l'abstrait par la métaphore ou la synecdoque constitue, on le sait, une faculté déterminante de toute activité intellectuelle.

\section{LE MONT KAILAS, CENTRE DE L'UNIVERS?}

Dans la plupart des pays d'Asie, un grand sommet a été identifié au mont Meru : en Chine le K'ouen-louen (et le Kouei-chan), à Java le mont Penaggungan, à Bali le mont Agung, au Japon le Fujiyama, etc. En Himalaya, la Montagne cosmique de la mythologie hindoue et bouddhiste est devenue le Kailas (Kailâsa). Cette montagne, située au-delà de la HauteChaîne, dans le Tibet occidental, à $1280 \mathrm{~km}$ à l'ouest de Lhasa, et à la verticale de la province indienne de Kamaon, est vénérée aussi bien par les hindous que par les bouddhistes. Dominant les vastes étendues désertiques des hauts plateaux tibétains de ses $6714 \mathrm{~m}$, le Kailas se dresse, majestueux, suffisamment bien séparé des autres sommets pour qu'on puisse en faire le tour facilement. Sa forme est pyramidale avec quatre arêtes bien découpées. Il porte sur sa face sud des stries noirâtres dans lesquelles l'imagination populaire reconnaît tantôt un svastika, marque bouddhiste de bon augure en forme de croix gammée ${ }^{(16)}$, tantôt les yeux du dieu Śiva. A ses pieds, s'étend le lac Manasarovar (330 km2), tache bleu turquoise, qui communique par un étroit chenal avec un autre lac plus petit : le Raksas Tal (ou Ravana Hrada) ${ }^{(17)}$. Le Manasarovar est situé à $4490 \mathrm{~m}$ d'altitude.

Kailas veut dire "Le Cristallin»; le mot vient du sanskrit kelasa, « cristal ». En tibétain, on l'appelle Kang Rinpoche, «Joyau des neiges » ou encore Ti-sé, « Le Pic ». Pour les hindous, cette montagne est le lieu de résidence de Śiva et de Pârvatî, ainsi que de Kubera, le fils du couple divin. Elle a en réalité deux significations différentes selon le degré d'avancement spirituel du fidèle. Au premier niveau (exotérique), le Kailas est le viśvalinga, le lïnga(=Śiva) du monde fiché dans un yoni, symbole de l'organe sexuel féminin. Mais ceux qui approchent de la libération finale voient dans cette montagne la lumière divine, autrement dit la vérité ultime ${ }^{(18)}$.

(16) Une conception tibétaine populaire figure un svastika debout sur la Montagne cosmique. La « montagne du svastika » a neuf étages, comme le Ciel et comme l'échelle qui y mène. Cf. R. A. STEIN, op. cit., p. 98.

(17) Selon une croyance tibétaine rapportée par E. KAWAGUCHI (Three years in Tibet, Kathmandu, Ratna Pustak Bandhar, 1909,p. 147, rééd. 1979), ces deux lacs sont comme mari et femme. Tous les quinze ans, le lac Raksas va rejoindre son épouse, le Manasarovar.

(18) Cf. R. SiNGH, « Pilgrims return to Kailas, Tibet's sacred mountain », Smithsonian, May 1982, pp. 94-100 (ici p. 100). 
Chez les Tibétains, plusieurs divinités ou héros semi-divins sont venus s'agréger à ce mont sacré. Le Kailas symbolise d'abord et avant tout le séjour du Bouddha, l'Illuminé, entouré de ses 500 bodhisattava. C'est également la demeure de la divinité protectrice Demchog. (appelée aussi Paro) et de son épouse Dorje-Phagmo (=Vajra Vârâhî). Les Tibétains situent aussi à cet endroit le lieu où Padmasambhava défit les tenants de la vieille religion du Bon, eux et leur chef Narabon chung, imposant par là-même le bouddhisme au Tibet. Les traces de ce combat sont encore visibles, dit-on, sur l'une des faces de la montagne ${ }^{(19)}$. Ils associent par ailleurs au Kailas le grand yogi Milarepa qui, après avoir passé le plus long de sa vie dans le Tibet occidental, mourut au XIe siècle juste au-dessus du lac Manasarovar.

Cette montagne est donc chargée d'une signification religieuse toute particulière. Pour les bouddhistes comme pour les hindous, les Indiens comme les Népalais, c'est l'un des lieux de pèlerinage (tîrtha) les plus réputés de tout l'Himalaya ${ }^{(20)}$. Sur place, les pèlerins font le tour (parikrama en sanskrit) du mont Kailas - $50 \mathrm{~km}$ environ - en passant par cinq monastères bouddhistes du lieu. En règle générale, les pèlerins ne font qu'une seule circumambulation. Mais les Tibétains orthodoxes peuvent en faire successivement de trois à treize et les plus pieux font le tour du Kailas en s'allongeant par terre de toute la longueur de leur corps à chaque pas : à ce rythme, ils mettent quinze jours pour achever la circumambulation complète. Lorsqu'il s'agit d'assurer le salut de l'âme d'un parent récemment décédé, les personnes aisées emploient des gens pauvres, moyennant rémunération, pour faire le pèlerinage à leur place. Les membres de la vieille religion tibétaine Bon pré-bouddhiste font eux aussi le pèlerinage au Kailas, mais, pour se distinguer, ils effectuent le tour de la Montagne sacrée dans le sens contraire des sectes bouddhistes, c'est-à-dire de la gauche vers la droite ${ }^{(21)}$. Les mérites que l'on tire de ces marches giratoires sont appréciables : un tour du Kailas efface, pense-t-on, les péchés d'une vie, dix tours gomment les péchés d'une ère cosmique, kalpa, cent huit tours procurent à l'individu la libération, le nirvâna, dans cette vie même ${ }^{(22)}$.

Le lac Manasarovar (en tibétain: Tso Mapham ou Tso Marang) dans lequel se mire la montagne est également renommé : c'est l'un des lacs les plus sacrés de tout l'Himalaya. Les pèlerins en font le tour en deux jours, choisissant le moment de l'année où le lac est gelé, sinon les berges sont difficilement praticables. Les mérites qu'on peut y obtenir sont immenses : l'hindou qui s'y baigne gagne le paradis de Brahmâ ; celui qui boit son eau se voit directement transporté dans le séjour céleste de Śiva et est lavé des péchés commis au cours de ses cent dernières vies. L'animal qui porte le nom de Mânasa sera, lui-même, promis au paradis de Brahmââ(23).

(19) R. DE NEBESKY-WOJKOWITZ, Oracles and demons of Tibet, Graz (Autriche), Akademische Druck-u Verlagsanstalt, 1975 (1 re édit. : 1956), p. 223.

(20) Depuis la guerre sino-indienne de 1962, les Indiens n'ont plus le droit de faire le pèlerinage au mont Kailas. Cependant, depuis 1982 les autorités chinoises admettent chaque année un petit contingent de pèlerins indiens; cf. R. SINGH, op. cit.

(21) Cf. NEBESKY-WoJKOWITZ, op. cit., p. 223.

(22) La plupart de ces données sont tirées du livre du Swami Pranavananda, Kailas Mânasarovar, New Delhi, Surya Print Process, 1983 (republication de Exploration in Tibet, même auteur, Calcutta, University of Calcutta, 1949), qui reste encore aujourd'hui un document de travail inégalé sur cette montagne sacrée.

(23) Cf. C. Allen, citant le Mahâbhârata (C. ALLEN, A Mountain in Tibet. The search for Mount Kailas and the sources of the great rivers of India, London, André Deutsch, 1982, p. 26). 


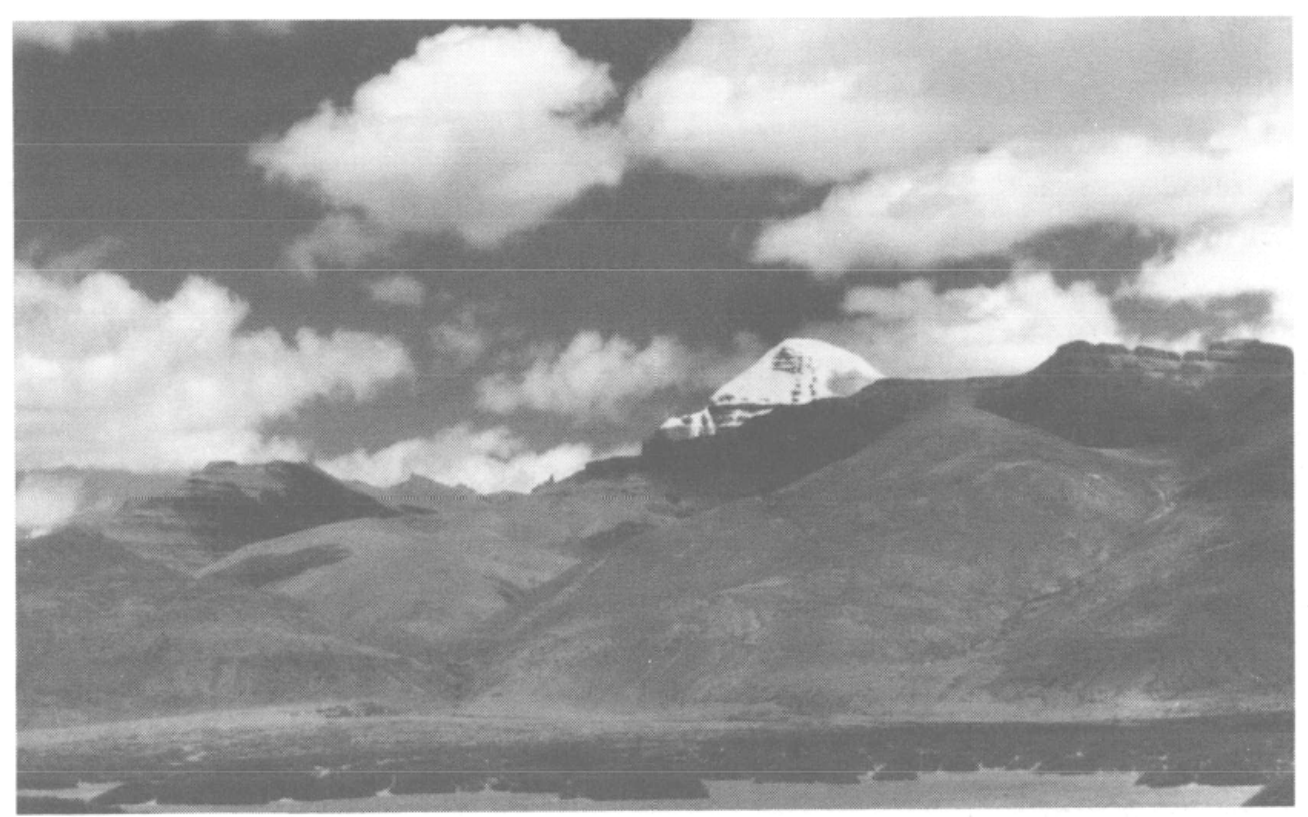

Un guide de pèlerinage tibétain composé à une date indéterminée, le Kangri Karchak (dgar-chag), expose les conceptions tibétaines relatives au Kailas. Ce mont, est-il écrit, se dresse au centre de l'univers tout entier, il s'élance vers le ciel, droit « comme la poignée d'un moulin à grain »(24). A mi-hauteur, sur un côté, pousse l'arbre kalpa-vrkșa, qui exauce tous les vœux. Les quatre faces de la montagne sont chacune d'une matière précieuse différente : la face Est est en cristal, la face Sud en saphir, celle de l'Ouest en rubis, et celle du Nord en or. Sur le sommet, couvert d'arbres chargés de fruits et de fleurs odoriférantes, vivent le dieu Demchok et sa parèdre Dorje-Phagmo, entourés de cinq cents bodhisattva. C'est là que les divinités régnant sur les 28 maisons lunaires, nakșatra, furent soumises par Padmasambhava. C'est là également qu'on peut entendre le son d'instruments de musique liturgique tels les cymbales et les cloches. A la base de la montagne, sur les quatre côtés, des empreintes des pieds du Bouddha sont inscrites dans le sol : elles maintiennent le Kailas fermement ancré à la terre. Aux quatre coins on trouve aussi des chaînes qui rivent la montagne au sol et empêchent les esprits néfastes de la tirer vers les mondes souterrains.

Ce texte fournit également de précieuses indications sur le Manasarovar. Le lac contiendrait dans ses eaux profondes un château habité par le roi des Nâga. Au milieu s'élèverait un arbre, invisible aux humains : en tombant ses fruits feraient le son jam ; c'est la raison pour laquelle la terre qui s'étend alentour s'appelle Jambuling (=Jambudvîpa). Certains des fruits qui tombent dans le lac sont mangés instantanément par les Dieux-serpents Nâga, d'autres sombrent au fond et se transforment en or. Cet arbre fait penser au lotus qui, dans maintes mythologies bouddhistes et hindouistes
Le mont Kailas, face Sud. Photo J. MANSION.

(24) On retrouve ici le mouvement tournant associé à la montagne sacrée. 
de la région, surgit de l'Océan originel ${ }^{(25)}$. C'est de ce lotus, on s'en souvient, qu'est né le Bouddha. Dans les écritures pâlies du bouddhisme, le Manasarovar est appelé Anavațapța ( le lac placide », " sans trouble »). Ce serait le seul paradis sur terre. A sa surface fleurissent des fleurs de lotus géantes où Bouddha et bodhisattva se tiendraient assis (26).

Retrouvant les vieilles images védiques, le Kangri Karchak rapporte par ailleurs qu'un fleuve prend sa source au sommet du Kailas. Ce fleuve coule jusqu'au Manasarovar puis se divise en quatre grandes rivières, lesquelles, après avoir fait le tour du mont et du lac, s'en vont chacune dans une direction différente de l'univers. La première rivière, Langchen Khambab (" rivière sortant de la bouche d'un éléphant ») ou Gañgâ, coule vers l'ouest. La deuxième, Mapcha Khambab («rivière sortant du bec d'un paon ») ou Sindu, coule en direction du sud. Le troisième, Tamchok Khambab ( rivière sortant de la bouche d'un cheval ») ou Pakshu (Vakshu), se dirige vers l'est. La quatrième enfin, Senge Khambab (« rivière sortant de la gueule d'un lion ») ou Sita, se fraye un chemin vers le nord ${ }^{(27)}$. Ces rivières représenteraient respectivement la Sutlej, la Karnali (qui se jette dans le Gange, au niveau de Patna, dans la plaine indienne), le Brahmapoutre et l'Indus. Les eaux de la Sutlej seraient froides et contiendraient de la poudre d'or, celles de Karnali seraient chaudes et riches en argent, celles de Brahmapoutre seraient froides et regorgeraient d'émeraudes, celles de l'Indus seraient chaudes et contiendraient des diamants(28). Il est dit également que ceux qui boiront de l'eau de la première rivière deviendront forts comme des éléphants, ceux qui boiront l'eau de la deuxième rivière deviendront beaux comme des paons, ceux qui boiront de l'eau dans la troisième deviendront vigoureux comme des chevaux. Quant à ceux qui boiront l'eau de la dernière rivière citée, ils deviendront héroïques comme des lions ${ }^{(29)}$.

La Montagne cosmique et le lac sont presque toujours associés dans les mythologies tibétaines et chinoises. Ils sont perçus comme complémentaires. La montagne, associée au Haut, au Feu et au Nord-Ouest, s'oppose au lac lié, lui, au Bas, aux Eaux et au Sud-Est ${ }^{(30)}$. Ce thème réapparaît parmi les populations de langue tibéto-birmane du Népali(31).

(25) Cf. par exemple G. TOFFIN (op. cit., p. 52) pour la vallée de Kathmandou. Les Tamang, une ethnie de langue tibéto-birmane du Népal, racontent : « Au début, l'univers est vide, il n'y a ni ciel, ni terre; en bas, la terre est recouverte d'un lac immense. Au milieu, le Dorje Gyadam, le spectre de l'univers, repose. La première forme qui en sort est le dieu Mathu Kaitap (Visnu), associé au démiurge Madan Rudra. Du nombril de Mathu Kaitap sort l'arbre de lotus, et au sommet de l'arbre de lotus apparaît le guru Pema » (B. STEINMANN, Les Tamang du Népal. Usages et religion, religion de l'usage, Paris, Ed. Recherche sur les Civilisations, A.D.P.F., 1987, p. 198). Le thème du lotus comme arbre de vie surgissant des Eaux primordiales et symbole de l'univers est profondément hindou ; cf. F.D.K. BOSCH (op. cit., ch. III).

(26) E. KAWAGUCHI, op.cit., p. 142.

(27) Swami Pranavananda (op. cit., p. 14). Cf. aussi C. Allen (op. cit., pp. 29-30).

(28) Swami Pranavananda (op. cit., p. 16).

(29) Swami PRANAVANANDA (op. cit., p. 17).

(30) R.A. STEIN, op. cit., p. 200.

(31) Par exemple chez les Tamang. Le mythe d'origine du monde de cette population fait état d'un lac originel (Mapham) et d'une montagne cosmique primordiale (Rigyal Lonbo); cf. B. STEINMANN (op. cit., pp. 198 et 223). 
Les textes hindous font eux aussi de fréquentes allusions au Kailas (Kailâsa) et au lac Manasarovar. Le Mahâbhârata et le Râmâyaṇa, les deux - grandes épopées indiennes, citent le Kailas comme une Montagne sacrée, mais ne l'identifient pas encore au mont Meru qui serait situé plus au nord. Ils le décrivent comme le lieu de résidence de Siva, Pârvatî et Kubera. A son sommet se dresserait un grand jujubier (sanskrit: badara), Zizypbus jujuba, au pied duquel le Gange prend sa source ${ }^{(32)}$. Les cinq frères Pânḍava visitèrent le Kailas pendant leur exil dans la forêt (Mahâbhârata, II).

Le lac Manasarovar (=Mânasa) est aussi mentionné dans les épopées, et le Skanda Purâna retrace son origine. Les fils de Brahmâ partirent, dit ce texte, vers le nord de l'Himachala pour accomplir des austérités sur le mont Kailas. Là, ayant vu Siva et Pârvatî, ils restèrent douze ans absorbés dans la prière et les mortifications corporelles. Le manque d'eau et de pluie leur rendait cependant la vie particulièrement précaire. Ils implorèrent Brahmâ de leur venir en aide. Brahmâ exauça leur vœu et, grâce à un effort de concentration mentale (manas), créa le lac Mânasa. Ses fils purent ainsi continuer leur ascèse, vénérant le Kailas et le lïnga doré qui s'élevait au milieu des eaux du lac (33).

Par la suite, le mont Kailas prit une importance accrue dans les textes hindous. On le décrivit de plus en plus comme un linga (de Śiva) - autre axe cosmique - et on l'assimila progressivement au mont Meru. Cette identification fut facilitée par les correspondances que la pensée hindoue établit entre les différents éléments et classes d'attributs qui composent l'univers, entre le macrocosme englobant et le microcosme directement observable par les individus. Ce souci de conformité et de corrélations que sous-tend l'idée d'une totalité organique du cosmos constitue, on le sait, une caractéristique de la pensée religieuse asiatique en général. Le Kailas devint alors une des multiples matérialisations du mont Meru qui, du templemontagne au linga de Śiva, du pilier de la maison à certains arbres sacrés, servent de support à la méditation et aux croyances des fidèles.

Mais pourquoi le Kailas? Pourquoi justement lui? Pourquoi cette montagne en vint-elle à surpasser en religiosité et sainteté tous les autres sommets de la chaîne himalayenne, eux aussi sacrés à divers titres? Beaucoup de points restent obscurs dans l'histoire de cette sacralisation, mais on peut sans risque avancer les remarques suivantes. La position géographique du Kailas, au-delà de la Haute-Chaîne, a été très certainement prise en considération par les populations locales. On se rappelle que les textes hindous situaient le Meru à trois chaînes de montagne de la plaine indienne, ce qui correspond grosso modo à la réalité topographique du Kailas. Le rapport n'a pas manqué d'être fait par les Indiens, toujours prêts à trouver confirmation de leurs textes sacrés dans la réalité quotidienne. Les particularités du réseau hydrographique de la région du Kailas ont dû, elles aussi, jouer un rôle. C'est dans cette zone en effet, dans un rayon de cent

(32) Cf. V. Mani, Puranic Encyclopaedia, Delhi, Motilal Banarsidass, 1975, p. 365, et C. ALLEN, op. cit., p. 23.

(33) Cité par C. AlleN (op. cit., p. 26). 
kilomètres autour de la montagne, que quatre grands fleuves prennent leur source: le Tsangpo, l'Indus, la Sutlej et la Karnali, un des principaux affluents du Gange. Les sources du Gange elles-mêmes ne sont qu'à $260 \mathrm{~km}$ vers le sud et l'on a longtemps cru, en Inde, qu'elles étaient reliées au lac Manasarovar par un chenal souterrain ${ }^{(34)}$. Les pèlerins et voyageurs, de plus en plus nombreux à circuler autour du Kailas à partir des XII-XIII siècles, le long de la grande route de commerce du Ladakh à Lhasa qui passe juste au nord, ont pu observer à loisir ce phénomène hydrographique. Ils ont dû être saisis par la présence à cet endroit, sur un périmètre restreint, des sources des quatre plus grands fleuves de l'Asie centrale et de l'Asie du Sud. Se souvenant du vieux mythe hindou du Meru, ils ont alors identifié le Kailas tibétain à la montagne cosmique. Jusqu'au début du $\mathrm{XIX}^{\mathrm{e}}$ siècle, l'imagerie indienne dominante situait d'ailleurs l'origine de ces quatre grands fleuves au mont Kailas(35). Il a fallu attendre les expéditions anglaises dans le Haut Himalaya au XIXe siècle pour que les sources du Tsangpo, de la Sutlej et de l'Indus soient reconnues avec précision et que le raccordement souterrain du Gange au lac Manasarovar apparaisse bel et bien comme une légende - du moins pour les Indiens les plus occidentalisés.

La permanence, sur près de trois mille ans, du symbolisme de la Montagne cosmique dans les mondes indien et tibétain est un trait qui ne peut manquer de frapper. Malgré les brassages de populations, les conquêtes, les évolutions économiques et sociales, les transformations des systèmes religieux, une indiscutable continuité se fait jour entre l'ancienne mythologie hindoue relative au Meru et les conceptions dont les Tibétains et les Indiens entourent présentement le mont Kailas. Les thèmes canoniques codifiés par écrit dans les textes sacrés ont traversé les siècles sans modification notable. Cette continuité se retrouve au plan synchronique dans la vaste aire géographique couverte par ces représentations. Comme on l'a vu, l'idée d'une Montagne cosmique, pilier du Monde et de la voûte céleste, déborde largement des domaines indien et tibétain : elle s'étend à tout l'Extrême-Orient et à l'Asie du Sud-Est. La puissance des idées hindoues, relayées sans doute par de vivantes traditions locales, a contribué à homogénéiser sur ce point précis mythologies et pratiques religieuses. Loin de nous l'idée de nier les variations à partir de mêmes thèmes fondamentaux et les déformations que ces notions ont pu subir ici et là.

(34) Sur cette question, voir le livre de C. ALLEN, $A$ mountain in Tibet. Le thème du chenal souterrain entre deux points d'eau existe ailleurs en Himalaya: pour la vallée de Kathmandou, cf. par exemple A.W. MACDONALD ( Notes népalaises : le Janaipurnimã et le Gosāikuṇa », in Langues et Techniques, Nature et Société, Ed. par J. M. C. THOMAS et L. BERNOT, Paris, Klincksieck, 1972, tome II, Pp. 73-81, qui le signale à propos du lac de Gosāikunda et des sources de la Trisuli Gandaki.

(35) Cf. par exemple E. KaWAGUCHI (op. cit., p. 141). Ce pèlerin japonais qui a séjourné dans le Tibet oriental à l'aube du $\mathrm{XX}^{\mathrm{e}}$ siècle qualifie le Kailas de « mandala naturel » (idem, pp. 137 et 141). 


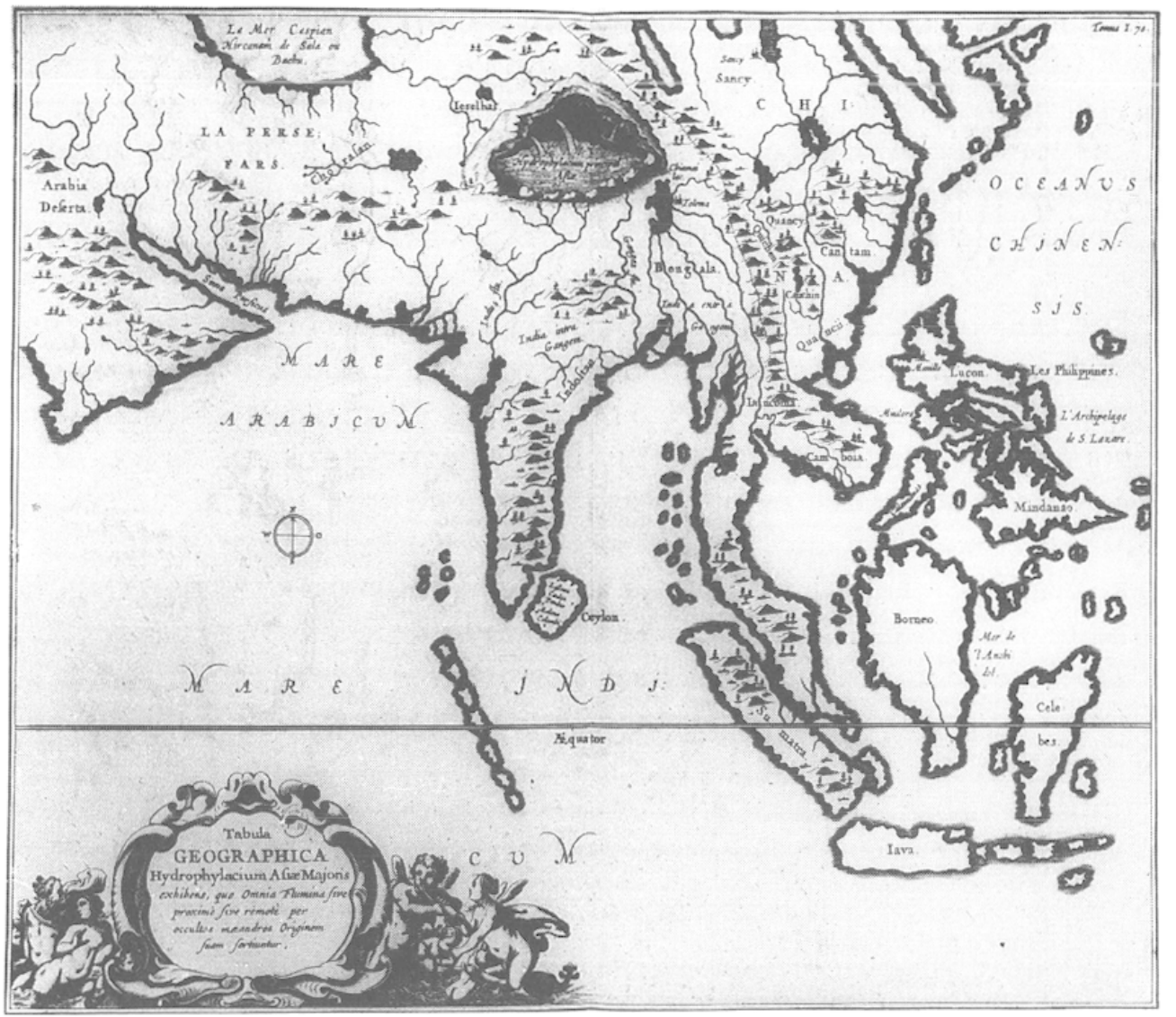

Carte de l'Asie, montrant les itinéraires de différents voyageurs européens. Tiré de l'ouvrage d'Athanasius Kircher: China monumentis qua sacris qua profanis nec non variis naturae et artis spectaculis, aliarumque rerum memorabilium argumentis illustrata..., Amstelodami, apud J. Janssonimu a Waesberg et $E$. Weyerstraet, 1667 ; édition française : La Chine d'Athanase Kirchere..., traduit par F.S. Dalquié. Amsterdam, J. Jansson et les héritiers de $E$. Weyerstraet, 1670 [Phot. Bibl. Nat. Paris].

Les documents occidentaux du XVIIe fournissent de précieux renseignements sur les représentations associées aux montagnes en Asie. Dans le livre susmentionné, A. Kircher, un jésuite du Würzburg, relate les pérégrinations en Haute Asie des peres $A$. Gruber et A. Dorville entre 1661 et 1662. C'est sur la foi des descriptions de ces deux pères, des jésuites eux aussi, que la carte reproduite ci-contre a été dessinée. Les sources du Gange et de I'Indus sont localisées ici au sommet d'une grande montagne du Tibet occidental, sans doute le Kailas. Au sud de cette montagne, est disposé un lac d'où naissent quatre grandes rivières de I'Inde. De toute évidence, motifs mythologiques hindous et relevés topographiques se mêlent ici étroitement, sans oublier peut-être des réminiscences bibliques.

Carte de l'Asie, tirée d'un ouvrage d'Athanasius Kircher; Mundus subterraneus. Amsterdam, Janssen-Waesberg, 1678 (1re édit.: 1665) [Phot. Bibl. Nat. Paris]. La carte montre le "réservoir d'eau (hydrophylacium) de l'Asie Majeure, dont tous les fleuves de l'Asie seraient de près ou de loin dérivés, se divisant de là par des sinuosités cachées. Cet hydrophylacium est localisé dans une caverne, da l'intérieur d'une grande montagne du Tibet. Nul doute que des images indiennes et tibétaines ont là aussi joué. Ce n'est qu'au XIXe siècle que les sources exactes des grands fleuves asiatiques ont été reconnues. [Je remercie L. Boulnois, du Centre d'études himalayennes, Meudon, d'avoir attiré mon attention sur cette carte]. 
Mais de telles divergences n'excluent pas une réelle cohérence, qu'une histoire des emprunts et des contacts devrait, un jour, permettre d'expliquer.

Valéry se demandait si nos abstractions n'avaient pas pour origine telle ou telle expérience personnelle et singulière, et si les notions d'infini, de profondeur, de connaissance, considérées aujourd'hui comme des catégories fondamentales de l'entendement humain, n'avaient rien à voir avec la présence d'une lumière, d'une étendue, d'une mobilité observées dans un élément du monde naturel : ciel, soleil ou mer ${ }^{(36)}$. Est-il totalement absurde de penser que la montagne, grande réalité élémentaire de la nature, ait pu imposer à l'homme l'idée d'une puissance transcendantale, suréminente, et celle d'un pont vers le ciel? Entre Hermogène et Cratyle une voie réconciliatrice est-elle possible? Questions plus philosophiques que scientifiques sans doute, mais qu'un spécialiste de l'histoire des religions ne saurait esquiver. En termes connexes, nous avons suggéré ici que la pensée mythique hindoue s'était inspirée de la présence de l'Himalaya à sa frontière septentrionale et qu'elle avait fait de cette massive barrière un élément important de sa géographie religieuse : la chaîne himalayenne a de toute évidence marqué les représentations purâniques. Dans un deuxième temps, nous avons montré que la pensée mythique avait également projeté des notions déjà constituées sur ces montagnes et qu'elle avait en quelque sorte sanctifié le Kailas en l'identifiant au mont Meru. Les mécanismes à l'œuvre dans ce processus sont sans doute autrement plus complexes, mais un tel va-et-vient entre réel et imaginaire constitue à notre sens une caractéristique essentielle de la pensée symbolique: cette dernière subit autant l'empreinte du milieu physique qui l'entoure qu'elle « informe » ce milieu, le chargeant de sens et de contenu signifiant. Sujet et objet ne sont pas deux pôles fixes et séparés : ils s'élaborent et se modifient l'un à partir de l'autre. Cette sorte de dualité dynamique, de double mouvement d'appropriation et de déposition, autorise à voir dans le symbolique quelque chose qu'on pourrait qualifier de transitionnel.

Les religions des civilisations extra-européennes ont longtemps été considérées comme des ensembles disparates de superstitions nées de l'ignorance et soumises à l'arbitraire. Cette image est aujourd'hui complètement rejetée : les mythes et les conceptions cosmogoniques dont il a été question dans ces pages appartiennent à un système de pensée cohérent et unifié reliant l'homme au cosmos et à ses semblables selon des lois précises. Il existe dans de telles cultures d'étroites relations d'interdépendance entre les structures sociales et les faits religieux. Rites et croyances d'un côté, relations sociales et actes quotidiens de l'autre se donnent en même temps sans que le domaine des uns soit bien distingué de celui des autres. Tous les sensibles y sont des signifiants, tous les signifiants y sont sensibles. A la limite, la religion ne constitue pas ici une fonction différenciée. L'opposition entre sacré et profane subsiste, mais sous

(36) P. VALÉRY, « Inspirations méditerranéennes *, in Conférences, Paris, Gallimard, 1939, pp. 27-46 (repris dans Euvres, I, Paris, Bibl. de la Pléiade, 1957). 
d'autres formes, nettement moin tranchées que dans notre monde sécularisé.

Quelle est la nature des liens entre société et religion dans ces civilisations asiatiques? Ce fut l'erreur du fonctionnalisme de vouloir expliquer les aspects religieux en général en les déduisant de l'activité sociale. Le symbolisme possède son autonomie et fonctionne selon des lois propres qui ne doivent rien aux faits sociaux. Plus encore : c'est la vision symbolique elle-même qui bien souvent dévoile le monde et aide l'homme à se sentir accordé à l'universel. Les notions conceptuelles relatives à l'ordre du cosmos se révèlent cruciales pour comprendre la réalité sociale dans son ensemble. En Inde comme au Tibet, les schèmes religieux induisent des rituels, des pèlerinages, en un mot des pratiques qui imprègnent les gestes les plus élémentaires de la vie courante. De la même manière, les rites apparaissent souvent comme des mises en scène, des actualisations de conceptions symboliques. Une telle unité interne et l'intégration du vécu qu'elle soustend autorisent à faire des rapprochements qui, ailleurs, pourraient sembler hasardeux. Un exemple entre cent, qui nous ramène directement à notre sujet : la circumambulation qu'effectuent les fidèles hindous et bouddhistes autour d'éminences sacrées (trait caractéristique de l'attitude de ces populations à l'égard des montagnes) n'évoque-t-elle pas ce mouvement tournant dont les mythes et les architectes asiatiques font un des éléments essentiels du symbolisme du Mont cosmique (et de l'Arbre de Vie)?

Contrairement à la vision occidentale moderne où tout a été désacralisé, y compris les actes physiologiques élémentaires comme l'alimentation et la sexualité, la pensée hindoue et tibétaine ne dissocie pas les images des usages, ni la connaissance de l'action.

Gérard ToFFIN

Directeur de recherche

ER 299 du CNRS

Meudon 